\title{
The Lille apathy rating scale (LARS), a new instrument for detecting and quantifying apathy: validation in Parkinson's disease
}

\author{
P Sockeel, K Dujardin, D Devos, C Denève, A Destée, L Defebvre
}

J Neurol Neurosurg Psychiatry 2006;77:579-584. doi: 10.1136/jnnp.2005.075929

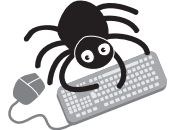

An appendix is available online at http://www. jnnp.com/supplemental

See end of article for authors' affiliations

a.................

Correspondence to: Dr Pascal Sockeel, UFR de Psychologie, BP 149, F59653 Villeneuve D'Ascq cedex, France; pascal. sockeel@univ-lille3.fr

Received 13 July 2005 In revised form

5 December 2005

Accepted 3 January 2006
Background: Apathy is usually defined as reduced interest and participation in various activities. It is a frequent consequence of neurological and psychiatric disorders. Although various scoring methods have been proposed, there is a lack of validated, standardised instruments for detecting apathy and assessing its severity.

Objective: To develop an apathy rating scale using a structured standardised interview capable of distinguishing between the condition's various features.

Methods: The Lille Apathy Rating Scale (LARS) is based on a structured interview. It includes 33 items, divided into nine domains. Responses are scored on a dichotomous scale. The participants used to validate the scale consisted of 159 patients with probable Parkinson's disease and 58 healthy control subjects. The Marin Apathy Scale, the Montgomery and Asberg Depression Rating Scale, and the Mattis Dementia Rating Scale were also administered.

Results: Principal component analysis showed that the LARS probed a single construct which forms the root of an oblique factor structure reflecting four dimensions: intellectual curiosity, self awareness, emotion, and action initiation. The main psychometric properties of the LARS (internal consistency, inter-rater and testretest reliability) were satisfactory. Concurrent validity was evaluated by reference to the Marin scale and to judgements provided by expert clinicians.

Conclusions: Standard validity indices showed that the LARS is sensitive and capable of distinguishing between apathy and depression. As a screening tool, the scale is able to support dichotomous judgements accurately and, when greater measurement sensitivity is required, also determine the severity of apathy within a four category classification.
A pathy refers to a set of behavioural, emotional, and cognitive features such as reduced interest and participation in the main activities of daily life, a lack of initiative, a trend towards early withdrawal from initiated activities, indifference, and flattening of affect. The concept of apathy lacks specificity and various different definitions have been suggested. For certain investigators, the key feature is lack of motivation, and apathy can thus be defined as "diminished motivation not attributable to diminished level of consciousness, cognitive impairment, or emotional distress". ${ }^{1}$ For others, the prime characteristic is lack of initiative: apathy is considered to be "an absence of responsiveness to stimuli as demonstrated by a lack of self initiated action". ${ }^{2}$ In addition to these conceptual difficulties, apathy overlaps with a range of other behavioural and psychological factors, including mood and some aspects of personality and cognitive function. ${ }^{3}$

The apathy evaluation scale (AES) proposed by Marin et al ${ }^{4}$ is currently the scale most often used to assess apathy. Its reliability and validity for measuring apathy in different pathological conditions have been demonstrated. ${ }^{4}$ However, the administration instructions and scoring method have suffered from a lack of standardisation. In clinical research and most drug treatment studies, apathy has been assessed with the Neuropsychiatric Inventory (NPI), which includes a specific item for the global evaluation of apathy. ${ }^{5}$ Recently, Robert et $a l^{6}$ proposed an extension of this assessment by using the Apathy Inventory, which allows separate assessment of emotional blunting, lack of initiative, and lack of interest in addition to the global NPI score. Despite the widely demonstrated validity of these latter scales, they are primarily dependent on the opinion of the caregiver, who may not always be available or reliable.

From a pathophysiological viewpoint, the most common cause of apathy is dysfunction of the frontal lobes, following either a direct lesion of the frontal cortex or damage to regions tightly connected to the latter (such as the basal ganglia). The frontal-subcortical circuits often seem to be involved, and apathy is a common behavioural consequence of basal ganglia disorders. ${ }^{237}$ Current estimates of the prevalence of apathy in Parkinson's disease vary from $16.5 \%$ to $42 \% .^{37^{8}}$ It is also frequently observed in other conditions with parkinsonism. ${ }^{9}$

Our aim was to develop an apathy rating scale using a structured, standardised interview capable of distinguishing between the various features of apathy. We examined its concurrent validity, internal consistency, and inter-rater reliability in a population of healthy subjects and patients with Parkinson's disease. The latter group was chosen in view of the fact that apathy is commonly observed in this basal ganglia degenerative disease. ${ }^{2}$

\section{METHODS}

\section{Participants}

One hundred and fifty nine patients with probable Parkinson's disease participated in the study (the demographic characteristics are shown in table 1). Parkinson's

Abbreviations: AES, apathy evaluation scale; DRS, dementia rating scale; LARS, Lille Apathy Rating Scale; MADRS, Montgomery and Asberg depression rating scale; NPI, Neuropsychiatric Inventory; UPDRS, Unified Parkinson's Disease Rating Scale 
Table 1 Demographic and clinical data for the subject groups

\begin{tabular}{llll}
\hline Variable & $\begin{array}{l}\text { Stable Parkinson's } \\
\text { disease }\end{array}$ & $\begin{array}{l}\text { Fluctuating Parkinson's } \\
\text { disease }\end{array}$ & $\begin{array}{l}\text { Parkinson's disease with } \\
\text { dementia }\end{array}$ \\
\hline Number of subjects (M/F) & $47(30 / 17)$ & $73(40 / 33)$ & $39(17 / 23)$ \\
Age (years) & $62.0(11.4)$ & $60.5(8.2)$ & $68.6(8.9)$ \\
Education duration (years) & $11.89(3.29)$ & $11.14(2.95)$ & $8.79(2.32)$ \\
Mattis DRS score (max=144) & $136.96(4.60)$ & $134.33(5.69)$ & $118.67(7.84)$ \\
MADRS score (max=60) & $6.85(6.09)$ & $9.76(6.66)$ & $11.05(6.17)$ \\
UPDRS score (max =108) & $15.53(8.53)$ & $26.92(12.56)$ & $32.46(14.71)$ \\
Dopa equivalent dosage (mg/day) & $711(400)$ & $905(506)$ & $912(507)$ \\
\hline
\end{tabular}

Values are mean (SD).

DRS, dementia rating scale; F, female; M, male; MADRS, Montgomery and Asberg depression rating scale; NA, not available; UPDRS, Unified Parkinson's Disease Rating Scale.

disease was defined according to the United Kingdom Parkinson's disease brain bank criteria. ${ }^{10}$ The study population was separated into three groups:

- 47 patients with non-demented Parkinson's disease early in the course of the disease and well stabilised on antiparkinsonian drug treatment (stable Parkinson's disease);

- 73 non-demented patients with fluctuating Parkinson's disease and severe symptoms (fluctuating Parkinson's disease):

- 39 patients with Parkinson's disease and a diagnosis of dementia according to the fourth Edition of the Diagnostic and Statistical Manual of Mental Disorders (DSM-IV) (Parkinson's disease with dementia).

Eighteen patients early in the course of the disease were not receiving any treatment at all. Sixty four patients were being treated with levodopa only (mean (SD) (range): levodopa equivalent dosage $=692.8(360.0) \mathrm{mg} /$ day $(100$ to $1700)$ ); 10 were receiving a dopamine agonist only (levodopa equivalent dosage $=487.0(461.7) \mathrm{mg} /$ day $(120$ to 1500$)$ ); and 67 were receiving levodopa in combination with a dopamine agonist (levodopa equivalent dosage $=1038.1$ (513.0) $\mathrm{mg} /$ day (150 to 2337.5)). Motor disability was evaluated using the motor score on the Unified Parkinson's Disease Rating Scale (UPDRS-III). ${ }^{11}$ Although none of the patients were suffering from any neurological disease other than Parkinson's disease, 24 (15\%) had a history of drug induced psychosis. Sixty six patients were receiving treatment for anxiety related, depressive, or psychotic symptoms (23 were on selective serotonin reuptake inhibitors only, nine were on alprazolam or bromazepam only, seven were taking mianserine only, 21 were taking alprazolam or bromazepam plus mianserine $(n=17)$ or a selective serotonin inhibitor $(n=4)$, and 10 were being treated with clozapine). Five patients were being treated with acetylcholinesterase inhibitors for their cognitive disorders.

Fifty eight healthy control subjects were chosen to match the patient group as closely as possible with respect to age and educational level (table 1).

None of the controls had a personal history of neurological or psychiatric illness.

All participants gave their informed consent to participation in the study.

\section{Evaluation scales}

Apathy was assessed with the Marin apathy evaluation scale (AES) and our new Lille Apathy Rating Scale (LARS), as described below. The scale and its instructions for use are shown in the appendix which can be viewed on the journal website (http://www.jnnp.com/supplemental).

Severity of depressive symptoms was assessed using the Montgomery and Asberg depression rating scale
(MADRS). ${ }^{12}$ In addition to the global score and in view of the scale's factorial structure, we also used three subscores for assessing dysphoric apathy, psychic anxiety, and vegetative symptoms. ${ }^{13}$

Global cognitive efficiency was assessed in terms of the global score (out of 144) on the Mattis Dementia Rating Scale (Mattis DRS). ${ }^{14}$

All participants underwent assessment of apathy, depression, and cognitive efficiency during a one hour test session.

\section{Scale structure, item development and selection}

The LARS was based on the main conceptual principles proposed by Marin $e t \mathrm{al}^{4}$ and on our own clinical experience. The scale includes 33 items, divided into nine domains. Eight of these concern the main clinical manifestations of apathy, as described in published reports: reduction in everyday productivity; lack of interest; lack of initiative; extinction of novelty seeking and motivation; blunting of emotional responses; lack of concern; and poor social life. The ninth domain (extinction of self awareness) refers to a particular manifestation of apathy highlighted by Stuss et al, ${ }^{2}$ who considered self and social awareness as "a metacognitive ability, necessary to mediate information from a personal, social past and current history with projections to the future". The main impact of apathy on this ability would be a reduction in self criticism and behavioural adjustment to social requirements in one's own interest.

The items are presented as positively worded questions to which the subject is expected to answer clearly "yes" or "no", in order to reduce subjective interpretations as much as possible. With the exception of the first three questions (which are coded on a five point Likert-type scale), responses are coded by the clinician on a binary (yes/no) scale, with an additional "NA" (not available) condition for non-classifiable answers or non-applicable items. Finally, the scale was designed in such a way that each of the nine domains can be evaluated through subscales which contribute with equal weighting to the global score. Hence the global score ranges from -36 to +36 , with a higher score representing a greater degree of apathy.

\section{Procedure}

The scale's rating is based on a subject's own report (during a structured interview) on their thoughts, emotions, and activities over the previous four weeks. In order to evaluate inter-rater reliability, simultaneous ratings on a subset of Parkinson's disease patients $(n=32)$ were carried out by two clinicians. Finally, a subset of patients $(n=35)$ participated in a retest procedure approximately four months later.

The Parkinson's disease patients were also examined by an independent group of clinicians. These were asked to judge (on the basis of a clinical examination and interview) whether or not the individual was depressed or apathetic or both. When a patient was categorised as apathetic, the 


\begin{tabular}{|c|c|c|c|c|}
\hline Variable & $\begin{array}{l}\text { Factor } 1 \\
\text { IC }\end{array}$ & $\begin{array}{l}\text { Factor } 2 \\
\text { SA }\end{array}$ & $\begin{array}{l}\text { Factor } 3 \\
\text { E }\end{array}$ & $\begin{array}{l}\text { Factor } 4 \\
\text { Al }\end{array}$ \\
\hline \multicolumn{5}{|c|}{ (A) Factor loadings } \\
\hline EP & 0.101 & 0.056 & 0.164 & 0.867 \\
\hline INT & 0.453 & 0.084 & 0.291 & 0.371 \\
\hline INI & 0.344 & -0.046 & 0.015 & 0.692 \\
\hline NS & 0.694 & 0.066 & 0.235 & 0.228 \\
\hline M & 0.719 & 0.066 & 0.086 & 0.200 \\
\hline ER & 0.129 & 0.386 & 0.700 & 0.135 \\
\hline $\mathrm{C}$ & 0.158 & -0.225 & 0.816 & 0.088 \\
\hline s & 0.790 & -0.024 & 0.031 & 0.065 \\
\hline SA & 0.058 & 0.931 & -0.003 & 0.008 \\
\hline \multicolumn{5}{|c|}{ (B) Correlations between oblique factors } \\
\hline IC & 1.000 & 0.126 & 0.435 & 0.565 \\
\hline SA & 0.126 & 1.000 & 0.112 & 0.031 \\
\hline $\mathrm{E}$ & 0.435 & 0.112 & 1.000 & 0.293 \\
\hline $\mathrm{Al}$ & 0.565 & 0.031 & 0.293 & 1.000 \\
\hline \multicolumn{5}{|c|}{$\begin{array}{l}\text { Numbers in bold type indicate the correlations that determine the clusters for the oblique factors in }(A) \text { and } \\
\text { significant correlations between oblique factors }(p<0.01) \text { in }(B) \text {. } \\
\text { Al, action initiation; } C \text {, lack of concern; } E \text {, emotion; EP, everyday productivity; ER, blunting of emotional responses; } \\
\text { IC, intellectual curiosity; INI, lack of initiative; INT, lack of interest; } M \text {, motivation; NS, extinction of novelty seeking; } \\
\text { SA; extinction of self awareness; SL, poor social life. }\end{array}$} \\
\hline
\end{tabular}

clinician had to describe their behaviour using one of the following three adjectives: "mild," "moderate," or "severe." Statistics and data analyses were carried out using Statistica 6.0 software.

\section{RESULTS}

\section{Factorial structure}

First, the 33 items were analysed in terms of potential intercorrelations. As expected, an exploratory examination of the correlation matrix showed relatively low correlations between the scores in items belonging to different domains (lowest coefficient $=-0.13$, highest coefficient $=0.36$, mean (SD) $r=0.10(0.10))$. The correlations between items belonging to the same domain were reasonably high (lowest coefficient $=0.12$, highest coefficient $=0.58$, mean $r=0.32$ $(0.17))$, whereas the correlations between scores for each item and for the scale as a whole were high (lowest coefficient $=0.52$, highest coefficient $=0.81$, mean $r=0.65$ (0.11)). Hence, these results support our choice of a procedure with dichotomous items which are then collapsed into subscales to improve sensitivity. Finally, and with the exception of self awareness $(r=0.28)$, the different subscales corresponding to the nine domains were highly correlated to the global LARS score (lowest coefficient $=0.45$, highest coefficient $=0.72$, mean $r=0.60(0.08))$.
Table 4 Sensitivity of the main scores and subscores of LARS and MADRS to apathy and depression, as determined by multivariate analysis of variance

\begin{tabular}{lrrl}
\hline & Depression & Apathy & Interaction \\
\hline LARS (main score) & $\mathbf{1 3 . 2 6}$ & $\mathbf{1 1 2 . 0 3}$ & 0.31 \\
Intellectual curiosity & $\mathbf{2 0 . 1 4}$ & $\mathbf{7 6 . 5 0}$ & 1.81 \\
Emotion & 0.99 & $\mathbf{1 6 . 2 5}$ & 0.40 \\
Action initiation & 1.32 & $\mathbf{3 2 . 4 2}$ & 0.52 \\
Self awareness & 0.77 & $\mathbf{6 . 5 8}$ & 0.85 \\
& & & \\
MADRS (main score) & $\mathbf{1 4 1 . 1 0}$ & $\mathbf{1 3 . 0 4}$ & 0.60 \\
Dysphoric apathy & $\mathbf{7 2 . 5 8}$ & $\mathbf{2 2 . 1 2}$ & 0.78 \\
Psychic anxiety & $\mathbf{6 6 . 6 6}$ & 1.31 & 0.00 \\
Vegetative symptoms & $\mathbf{2 4 . 6 4}$ & 0.35 & 0.01 \\
\hline
\end{tabular}

Bold type indicates significant $F_{(1,115)}$ values at $p<0.01$.

LARS, Lille Apathy Rating Scale; MADRS, Montgomery and Asberg depression rating scale.

An exploratory principal component analysis was carried out on the nine subscores in order to determine the structure of the data: four factors were identified with eigenvalues close to 1 , explaining more than $65 \%$ of the total variance. The first factor accounted for $34 \%$ of the total variance, confirming the one dimensional nature of the LARS.

\begin{tabular}{|c|c|c|c|}
\hline Patient group & n (\%) & LARS & MADRS \\
\hline No apathy or depression & $82(56.1)$ & $\begin{array}{l}-25.91(5.02) \\
{[-26.80 \text { to }-25.03]}\end{array}$ & $\begin{array}{l}5.52(3.29) \\
{[4.80 \text { to } 6.25]}\end{array}$ \\
\hline Apathy only & 37 (23.3) & $\begin{array}{l}-13.54(6.5) \\
{[-15.72 \text { to }-11.36]}\end{array}$ & $\begin{array}{l}7.95(3.93) \\
{[6.64 \text { to } 9.26]}\end{array}$ \\
\hline Depression only & $9(5.6)$ & $\begin{array}{l}-22.11(5.51) \\
{[-26.35 \text { to }-17.87]}\end{array}$ & $\begin{array}{l}15.00(5.38) \\
{[10.86 \text { to } 19.14]}\end{array}$ \\
\hline Apathy + depression & $31(19.5)$ & $\begin{array}{l}-8.35(8.35) \\
{[-11.41 \text { to }-5.30]}\end{array}$ & $\begin{array}{l}18.74(5.19) \\
{[16.84 \text { to } 20.65]}\end{array}$ \\
\hline
\end{tabular}

Values are mean (SD) and [95\% confidence intervals].

LARS, Lille Apathy Rating Scale; MADRS, Montgomery and Asberg depression rating scale. 
A further principal component analysis using oblique rotation generated a satisfactory model for this number of factors (goodness of fit with $\chi^{2}(\mathrm{df} 6)=9.69, \mathrm{p}>0.13$, NS). Table 2 gives the factor loadings and the correlations between the hierarchical structure's different components.

First, four clusters of variables were found, with either high unique factor loadings or low cross loadings. Next, the correlations between these four primary oblique factors yielded a set of two secondary factors, with the first showing evidence for a main construct representing apathy (factors 1 , 3 , and 4) and a second one dealing more with self awareness (factor 2). Finally, there was evidence to suggest that the four primary factors might represent distinct dimensions of apathy: (1) intellectual curiosity (lack of interest, novelty seeking and motivation, poor social life), reflecting low interest in novelty as well as a drop in the perceived need for knowledge; (2) self awareness; (3) emotion (blunting of emotional responses, lack of concern); and (4) action initiation (low everyday productivity, lack of initiative).

\section{Internal consistency, test-retest, and inter-rater reliability}

Internal consistency was determined both in terms of split half reliability and Cronbach's standardized $\alpha$ coefficients. The between-items and between-subscales $\alpha$ values were 0.80 and 0.74 , respectively. Split half reliability was 0.73 and reached 0.84 after correction with the Spearman Brown "prophecy formula". When evaluated on a subgroup of 35 patients, the test-retest correlation coefficient was 0.95 . Inter-rater reliability was checked using intraclass correlation calculated from the ratios of the different sources of variance. The latter were generated by an analysis of variance (ANOVA) on repeated measures, with the identity of the judge as a dependent variable. This ANOVA did not reveal any differences between scorings $(\mathrm{F}<\mathrm{l}, \mathrm{NS})$, and the intraclass correlation was high $(r=0.98)$. According to Nunally, ${ }^{15}$ these values correspond to high reliability.

\section{Separability between apathy and depression}

Parkinson's disease patients were divided into four groups according to the clinicians' binary classification of apathy and depression (table 3). The MADRS and LARS scores were compared in a two way multivariate analysis of variance (MANOVA), with the two classifications as categorical predictors. In a second MANOVA, we entered various dependent variables: the MADRS "dysphoric apathy," "psychic anxiety," and "vegetative symptoms" scores on the one hand, and LARS subscores from the four factorial analysis clusters (referred as intellectual curiosity (IC), self awareness (SA), emotion (E), and action initiation (AI)) on the other. We hypothesised that the main scores or the subscores, or both, would be differentially sensitive to their corresponding factors-that is, apathy or depression. If the two constructs were independent, we expected not to see an interaction effect.
As shown in table 4, both LARS and MADRS were generally sensitive to both apathy and depression, although the results for different subscales were not homogeneous.

The LARS "IC" factor was reactive to both variables, showing the dependence between these two constructs. Nevertheless, the mean scores observed for the different patient groups, the confidence intervals, and the absence of interactions between the apathy and depression factors all argue in favour of the LARS's ability to assess apathy independently of depression. Examination of the results for the "dysphoric apathy" factor in the MARDS supported this conclusion, as the correlation between the LARS and the MADRS mainly reflected the correlation between the LARS and the depression scale's apathy subscore.

\section{Concurrent validity}

Concurrent validity was assessed, first, by the correlation between the AES and LARS scores, and second, by comparison of the frequency distributions between the different cut off scores and expert judgements. We noted a strong correlation between the global scores for the AES and the LARS $(r=0.87)$. Close examination of links between Marin's scale and the four LARS factors established that the strongest correlations were found with the IC $(r=0.84)$ and AI dimensions $(r=0.65)$. The correlation between AES and the $\mathrm{E}$ dimension was $r=0.44$ and comparisons between the SA dimension and AES yielded $r=0.15$.

Criterion related validity was measured in order to obtain the best accuracy relative to an expert diagnosis. It was calculated from two kinds of cross tabulation tables, referring to either an expert dichotomous patient classification (moderately/severely apathetic $v$ non-apathetic/mildly apathetic) or a four class categorisation of disease severity (nonapathetic, mildly, moderately, or severely apathetic). Cut off scores for the LARS were derived empirically from the distributions of apathetic and control subjects, in order to separate the two sets and to avoid the inclusion of subjects in an inappropriate category (fig 1). An initial cut off score was set at 2.5 SD below the mean score of the control group. Thereafter, and depending on the user's choice in terms of specificity and sensitivity, three cut off scores were proposed, as shown in table 5 .

Comparisons with Marin's AES were provided for three cut off scores calculated using the same methodology. For a prevalence of $29.56 \%$, the cut off value of -16 seemed to be the best compromise, with a $\kappa$ of 0.79 and a sensitivity index of 0.89 , which are considered to represent excellent agreement. ${ }^{16}$ Finally, classification into four categories was checked against a corresponding distribution of patients with the cut off values of $[-36 ;-22]$ for non-apathetic and $[-21 ;-17],[-16 ;-10]$, and $[-9 ;+36]$ for slightly, moderately, or severely apathetic subjects, respectively. Again, these values were adjusted empirically in order to create classes with minimum overlap and to provide as much agreement as possible with the experts. Table 6 shows the corresponding contingency cross tabulation, from which we calculated an
Table 5 Criterion related validity, sensitivity, and specificity indices and cut off values for the AES and LARS scales, with respect to dichotomous expert judgments of apathy

\begin{tabular}{llllll}
\hline Scale & Cut off scores & Accuracy & к & Sensitivity & Specificity \\
\hline LARS & $\geqslant-17$ & 0.89 & 0.76 & 0.94 & 0.87 \\
& $\geqslant-16$ & 0.91 & 0.79 & 0.89 & 0.92 \\
AES & $\geqslant-15$ & 0.91 & 0.79 & 0.87 & 0.93 \\
& $>20$ & 0.85 & 0.65 & 0.81 & 0.87 \\
& $>21$ & 0.86 & 0.65 & 0.77 & 0.89 \\
& $>22$ & 0.84 & 0.61 & 0.68 & 0.91 \\
\hline
\end{tabular}

AES, apathy evaluation scale; LARS, Lille Apathy Rating Scale. 


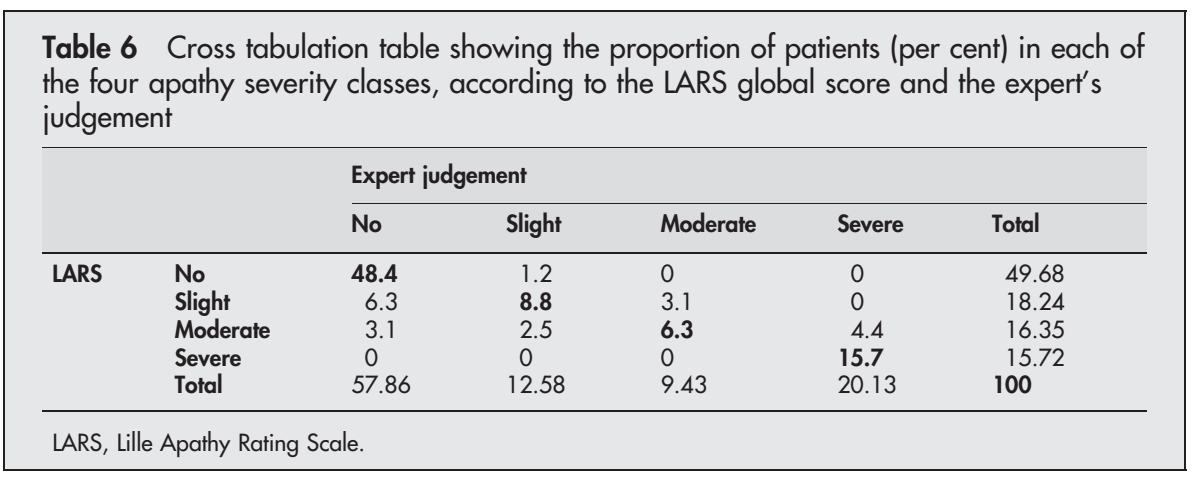

accuracy index of 0.81 with a $\kappa$ of 0.74 . These data clearly argue in favour of use of the LARS for optimally distinguishing between different degrees of severity in apathy syndromes.

\section{DISCUSSION}

This new Lille Apathy Rating Scale (LARS) was built on the conceptual basis of Marin's apathy evaluation scale (AES) but sought to remove various practical difficulties in using the latter. In particular, we have noticed first, a lack of standardisation in the administration and scoring procedures (because of the semistructured nature of the AES); second, variations in interpretation owing to multiple sources of informants; and third, possible sources of error because of fluctuation in the positive/negative orientation of questions. Moreover, we have also noticed a heterogeneous weighting of the items in relation to the main psychological domains. In order to enhance standardisation, improve stability, and reduce subjective interpretations during scoring, we adopted a dichotomous scale. Moreover, the nine domains of interest were equally weighted in the final score, enabling the generation of subject profiles. Question by question examination did not reveal floor or ceiling effects or extremely low or high inter-item correlations that would indicate incongruence or redundancy. This internal consistency was reinforced by relatively high reliability coefficients, suggesting that the scale indeed deals with a single, coherent construct.

In addition to the global assessment of apathy, the LARS also revealed a structure whose factors were interpreted as representing intellectual curiosity, action initiation, emotion, and self awareness. In fact, several studies 461718 have proposed definitions which incorporate distinct components

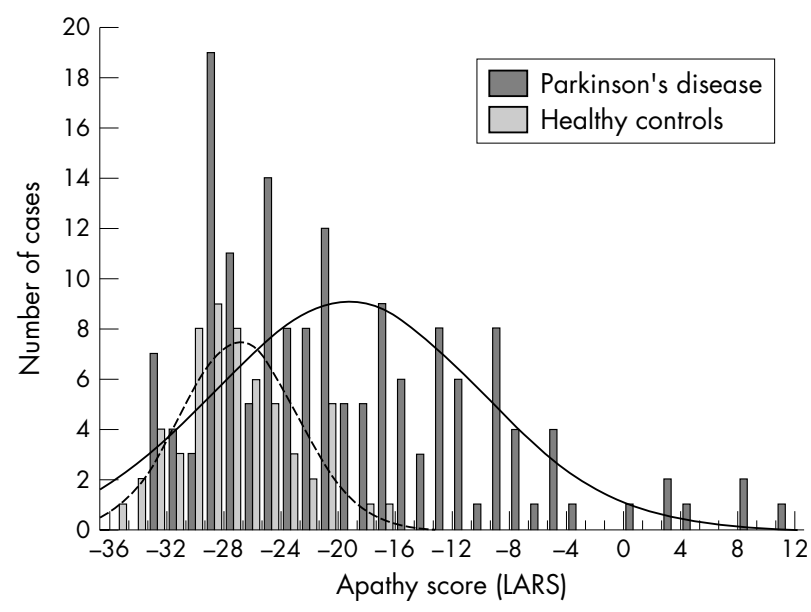

Figure 1 The distribution of LARS apathy scores for patients with Parkinson's disease and for healthy controls. of apathy (behavioural, cognitive, and emotional) and our results agree with and reinforce these suggestions, as these same three realms emerged from our analysis. Nevertheless, our data also generated a fourth factor, representing a reduction in self awareness and impaired behavioural adjustment to social life. This factor seemed to emerge in relation to certain specific characteristics of patients with cognitive decline.

Discriminating between depression and apathy has always been a tricky issue. Nevertheless, some studies have addressed this problem. For instance, Marin et al ${ }^{19}$ evaluated apathy and depression in patients with either Alzheimer's disease, stroke, or major depression. Although apathy and depression generally correlated within the groups, absolute scores varied considerably (and independently) between groups. Levy et $a^{20}$ found the same disease specific relation using the NPI and concluded that the presence of one condition did not predict the presence of the other. Pluck and Brown ${ }^{3}$ drew very similar conclusions: although the symptoms can dissociate within individual patients, comorbid depression and apathy appeared to have an additive effect on symptoms such as cognitive dysfunction. Our data seem to be compatible with published reports: the correlation between LARS and MADRS scores is clearly explained by the latter's dysphoric apathy subscale, which covaries with the LARS IC dimension (and, to a lesser extent, with AI). Moreover, the simultaneous presence of these two symptoms indicated the Parkinson's disease patients with the worst scores on the LARS. In practical terms, distinguishing apathy from depression implies that one should consider both the LARS and the MADRS scores: extremely low scores on both scales indicate patients with little suspicion of either condition; extremely high scores on both scales indicate patients with a high suspicion of both conditions; depressed patients should present a relatively high MADRS score with a relative low LARS score, whereas a relatively low MADRS score and a relatively high LARS score predict the presence of apathy in the absence of depression.

The main goal of this study was to provide a useful tool (meeting the usual psychometric prerequisites) for the assessment of apathy. We demonstrated that the LARS has very satisfactory inter-rater and test-retest reliability. Moreover, the establishment of excellent concurrent and criterion related validity was a major step in proving the value of LARS as a screening test. Furthermore, we proposed several cut off scores with good specificity and sensitivity. The comparison with Marin's AES criterion related validity was arduous, because we found several proposed AES cut off values. ${ }^{21} 22$ However, by opting for a cut off value corresponding to the mean AES score for the normal control group minus 2.5 SD, our sensitivity and specificity values showed that the LARS provided slightly more reliable validity. Finally, the four-class severity system may provide greater sensitivity by enabling precise measurements in treatment efficacy studies or, more generally, during patient follow up. 
In the current version of the LARS, all input information is obtained from the patient. In patients with anosognosia (who may thus underrate their symptoms), this may constitute a limitation. It is useful to obtain information from an informant in such cases, and we are currently working on an informant version of the LARS.

Our results showed that apathy is frequent in Parkinson's disease and that higher apathy levels are observed in patients with cognitive complications. The observed prevalence of $29 \%$ was within the range of previously reported values. ${ }^{378}$

\section{Conclusion}

The LARS is a reliable and practical instrument for assessing the multiple dimensions of apathetic syndrome. Its psychometric qualities appear to make it particularly suitable for assessing changes in the manifestations of apathy: in the future, the scale could thus constitute an interesting outcome variable for evaluating the efficacy of potential apathy treatments. Further studies will have to demonstrate the ability of the LARS to specify the apathy profile of different patient groups in relation to a given disease aetiology or severity level.

\section{Authors' affiliations}

P Sockeel, K Dujardin, Psychology Department, Charles De Gaulle University, Lille, France

D Devos, C Denève, A Destée, L Defebvre, Neurology and Movement Disorders Unit, EA2683, Faculty of Medicine and Lille University Hospital, Lille

Competing interests: none declared

\section{REFERENCES}

1 Marin RS. Differential diagnosis and classification of apathy. Am J Psychiatry 1990; 147:22-30.

2 Stuss DT, Van Reekum R, Murphy KJ. Differentiation of states and causes of apathy. In:Borod J, editor. The neuropsychology of emotion. New York: Oxford University Press, 2000:340-63.
3 Pluck GC, Brown RG. Apathy in Parkinson's disease. I Neurol Neurosurg Psychiatry 2002;73:636-42.

4 Marin RS, Biedrzycki RC, Firinciogullari S. Reliability and validity of the apathy evaluation scale. Psychiatry Res 1991;38:143-62.

5 Cummings JL, Mega M, Gray K, et al. The neuropsychiatric inventory: comprehensive assessment of psychopathology in dementia. Neuropsychology 1994;44:2308-14

6 Robert PH, Clairet S, Benoit $M$, et al. The apathy inventory: assessment of apathy and awareness in Alzheimer's disease, Parkinson's disease and mild cognitive impairment. Int J Geriatr Psychiatry 2002;17:1099-105.

7 Starkstein SE, Mayberg HS, Preziosi TJ, et al. Reliability, validity, and clinical correlates of apathy in Parkinson's disease. J Neuropsychiatry Clin Neurosci 1992;4:134-9.

8 Aarsland D, Larsen JP, Lim NG, et al. Range of neuropsychiatric disturbances in patients with Parkinson's disease. J Neurol Neurosurg Psychiatry 1999;67:492-6.

9 Litvan I, Mega MS, Cummings JL, et al. Neuropsychiatric aspects of progressive supranuclear palsy. Neurology 1996;47:1184-9.

$10 \mathrm{Gibb}$ WR, Lees AJ. The relevance of the Lewy body to the pathogenesis of idiopathic Parkinson's disease. J Neurol Neurosurg Psychiatry 1988;51:745-52.

11 Fahn S, Elton RL, and the members of the UPDRS Development Committee. The Unified Parkinson's disease rating scale. In:Fahn S, Marsden CD, Calne DB, Goldstein M editors. Recent developments in Parkinson's disease, vol 2. Florham Park, NJ: Macmillan Healthcare, 1987:153-63.

12 Montgomery SA, Asberg M. A new depression scale designed to be sensitive to change. Br J Psychiatry 1979;134:382-9.

13 Parker RD, Flint EP, Bosworth $\mathrm{HB}$, et al. A three-factor analytic model of the MADRS in geriatric depression. Int J Geriatr Psychiatry 2003;18:73-7.

14 Mattis S. Mental status examination for organic mental syndrome in the elderly patient. In:Bellak L, Karasy TE, editors. Geriatric psychiatry. New York: Grune and Stratton, 1976:77-121.

15 Nunally JC. Psychometric theory, 2nd edition. New York, NY: McGraw-Hill, 1978

16 Landis JR, Koch GG. The measurement of observer agreement for categorical data. Biometrics 1977;33:159-74.

17 Marin RS. Apathy: concept, syndrome, neural mechanism and treatment. Semin Clin Neuropsychiatry 1996;1:304-14.

18 Starkstein SE, Petracca G, Chemerinski E, et al. Syndromic validity of apathy in Alzheimer's disease. Am J Psychiatry 2001;158:872-7.

19 Marin RS, Firinciogullari S, Biedrzycki RC. Group differences in the relationship between apathy and depression. J Nerv Ment Dis 1994; 182:235-9.

20 Levy ML, Cummings JL, Fairbanks LA, et al. Apathy is not depression. J Neuropsychiatr Clin Neurosci 1998;10:314-19.

21 Andersson S, Krogstad JM, Finset A. Apathy and depressed mood in acquired brain damage: relationship to lesion localization and psychophysiological reactivity. Psychol Med 1999;29:447-56.

22 Kant R, Duffy JD, Pivovarnik A. Prevalence of apathy following head injury. Brain Injury 1998;12:87-92. 